\title{
The Role of Microblogging in OSS Knowledge Management
}

\author{
Jonathan Lewis \\ Hitotsubashi University, 2-1 Naka, Kunitachi-shi, 186-8601 Tokyo, Japan \\ jonathan_lewis@mac.com \\ http://www.lewis.soc.hit-u.ac.jp
}

\begin{abstract}
Given that microblogging has been shown to play a valuable role in knowledge management within companies, it is useful to understand how it is being used in relation to OSS. This project studies tweets related to 12 open source projects and keywords, ranging from web content management systems (CMSes) to general office applications. It found considerable differences in the content and exchange of tweets, especially between specialist products such as CMSes and office suites such as OpenOffice. Tweets concerning the more specialist projects tended to provide information rather than updates on the user's current status. We found a high proportion of event-driven traffic for some CMS projects, and a lower proportion for the office products and groups of projects.
\end{abstract}

Keywords: microblogging, twitter, knowledge management.

\section{Introduction}

In any knowledge-intensive project or organization, informal communication is vital to the timely spread of information and ideas. Considerable research has been undertaken on the role of microblogging services such as Twitter in knowledge management within enterprises [1-5]. Many OSS developers and users also use Twitter, but little attention has been paid to the role played by microblogging in exchanging and diffusing knowledge in OSS projects. This study of OSSrelated microblogging explores what kind of information is being exchanged on Twitter regarding open source software, and the different ways in which Twitter is being used. In order to answer these questions, a study was made of statuses (Tweets) related to 12 OSS projects, using a taxonomy adapted from previous research on intra-enterprise microblogging. The projects selected had an emphasis on, but were not restricted to, web content management. Table 1 gives an overview of the projects and the numbers of statuses collected, sampled and analyzed.

This paper is organized as follows: Section 2 discusses the selection of projects, presents statistics about the data used, and discusses the challenges in collecting and cleaning Twitter data. Section 3 contains the findings and analysis. Section 4 discusses threats to validity and topics for further research. 
Table 1. Overview of Twitter Statuses Retrieved and Sampled, 7 May-26 Dec 2012

\begin{tabular}{llrrr}
\hline Project/Keyword & $\begin{array}{l}\text { Project type } \\
\text { (language) }\end{array}$ & $\begin{array}{r}\text { Number of } \\
\text { statuses } \\
\text { collected }\end{array}$ & $\begin{array}{r}\text { Number } \\
\text { tagged } \\
\text { English }\end{array}$ & $\begin{array}{r}\text { Number } \\
\text { sampled }\end{array}$ \\
\hline Joomla & CMS (PHP) & 278,821 & 172,989 & 1,000 \\
TYPO3 & CMS (PHP) & 22,800 & 7,553 & 1,000 \\
SilverStripe & CMS (PHP) & 1,985 & 1,678 & 1,000 \\
Drupal & CMS (PHP) & 209,901 & 158,917 & 1,000 \\
Xoops & CMS (PHP) & 1,317 & 676 & 676 \\
Plone & CMS (Python) & 9,507 & 6,509 & 1,000 \\
RoR & Web app framework & 24,603 & 13,781 & 1,000 \\
& (Ruby) & 54,121 & 32,114 & 1,000 \\
PHP & Web scripting & & & \\
Apache & language & 28,3646 & 165,988 & 1,000 \\
Mozilla & Group of software & & & \\
Oprojects & Group of software & 30,9958 & 157,094 & 1,000 \\
libreoffice & projects & 35,718 & 11,185 & 1,000 \\
Sum & Office software & 12,905 & 1,969 & 1,000 \\
\hline
\end{tabular}

\section{$2 \quad$ Analyzing OSS Microblogging}

\subsection{Selection of Keywords/Projects}

Six web content management systems (CMSes) were selected, five of them written in PHP (Joomla, TYPO3, Silverstripe, Drupal, and Xoops) and one written in Python (Plone) 1 CMSes were selected because they are tightly focused on a particular product used by specialists (mostly website developers and system administrators) but nevertheless have large enough user and developer populations to generate sufficient traffic. Furthermore they can be compared to each other.

The server-side web scripting language PHP and the web application framework Ruby on Rails (RoR) were added in order to compare communications regarding web development using $\mathrm{PHP}$ and Ruby.

OpenOffice and libreoffice were included because, compared to the CMSes, they were likely to have more end-users who were not IT professionals. Their inclusion would thus help to highlight the characteristics of microblogging in the more specialist projects.

\footnotetext{
${ }^{1}$ Statuses were also gathered for the keywords 'Geeklog", 'Mambo", 'mojoPortal" and "WebGUI" in order to analyze the PHP-based content management system of those names, but the keywords were abandoned due to the small proportion of relevant statuses (in the case of Mambo) and the small number of statuses retrieved (in the other cases).
} 
Apache and Mozilla, two umbrella organizations for a number of open source projects, were included because, while being similarly Web-centered to the CMSes, their wider focus promised to show us differences between communication in individual projects and larger open source organizations.

\subsection{Selection of Microblogging Service}

While Twitter is the most well-known microblogging service, there is an alternative service, identi.ca, which uses open source software and, unlike Twitter, allows users to import and export their data using the FOAF standard. It might be the case that open source users and developers would make greater use of identi.ca for ideological reasons. In order to check whether this is the case, the search APIs of both services were queried for the selected keywords/projects between 15 February and 6 March 2013. The results are shown in Table 2. The results clearly show several orders of magnitude more activity regarding open source keywords on Twitter compared to identi.ca, which justifies the selection of Twitter as the data source for this study.

Table 2. Numbers of Statuses Retrieved from Twitter and identi.ca Search APIs, Feb 15-Mar 6, 2013

\begin{tabular}{lrr}
\hline Project/keyword & Twitter & identi.ca \\
\hline Joomla & 34,716 & 10 \\
TYPO3 & 2,422 & 0 \\
SilverStripe & 457 & 1 \\
Drupal & 24,591 & 65 \\
Xoops & 1,447 & 0 \\
Plone & 1,324 & 3 \\
RoR & 39,916 & 5 \\
PHP & 77,114 & 875 \\
Apache & 43,280 & 103 \\
Mozilla & 41,077 & 303 \\
OpenOffice & 4,160 & 21 \\
libreoffice & 6,567 & 357 \\
\hline Total & 277,071 & 1,743 \\
\hline
\end{tabular}

\subsection{Data Collection}

The Twitter Search API was queried approximately every two hours from 7 May to 26 December 2012 for keywords related to the 12 OSS projects. The keywords were not mutually exclusive, so for example a status containing both "PHP" and "Drupal" could be included in both samples 2 The statuses were saved to a PostGreSQL database. A total of 1,245,282 statuses containing the 12 keywords were collected, as shown in Table 1.

\footnotetext{
${ }^{2}$ In fact, 10 statuses occurred in samples for two keywords/projects.
} 
After collecting the statuses, a random sample of English-language statuses for each keyword was exported from the database for manual coding 3 A sample size of 1,000 statuses for each project was coded where available, giving a total of 11,676. This compares with the total of 3,152 Twitter posts examined by Ehrlich and Shami, although their sample was not divided into 12 projects.

\subsection{Data Cleaning}

The sampled data was cleaned by excluding the following kinds of status:

Non-English statuses. The number of non-English statuses in the sample was very low because the sample included only statuses tagged as English language.

Irrelevant statuses. The number of irrelevant statuses was also generally very low, with the exception of Apache, Mozilla and RoR. "Apache" obviously has many other uses, while "RoR" refers not only to Ruby on Rails but also to such things as the Retraining of Racehorses. There were a large number of irrelevant statuses containing the word "Mozilla" because when Firefox users tweeted titles of web pages on any topic the text would include "Mozilla Firefox."

Robot statuses. Many studies of microblogging have focussed on user behavior and accordingly have selected users who were recognizably individual people. Considerable numbers of Tweets, however, are generated automatically. This is also the case where open source software is concerned, and it is a thorny question whether to include them or not. It was decided to exclude these "robot" statuses, above all because they were much more numerous for some keywords/projects than others, making it difficult to compare results. Defining and identifying automatically generated statuses is not easy, but the following categories of statuses were excluded:

1. Machine status Tweets e.g.

Fedora [f18-arm] :: [97.44\%] Completed - [0] Built - [3] Failed :: Task

Error [perl-OpenOffice-UNO-0.07-3.fc17]-[1142021]

2. Repository-generated statuses e.g. cesag committed revision 1694 to the Xoops France Network SVN repository, changing 1 files: cesag committed revi... http://t.co/nXREVkxj

3. CMS change statuses generated by crawlers detecting changes in web page code e.g.

http://t.co/YhTLHvCe: Change from NetObjects Fusion to TYPO3 http://t.co/YhTLHvCe \#cms

4. Statuses sent automatically when someone posts to a forum e.g.

XOOPS: Re: Linux Xoops white page issue? [by kidx] http://t.co/MxW4r2Ba

5. Statuses generated by job sites. Based on examination of the samples, these were defined as posts with 'job' or 'elance' (from 'freelance') in the sender's name.

Duplicate statuses. Duplicate statuses, which were defined as those containing identical text (with any urls excluded) and posted by the same user. Statuses containing identical text but sent by different users were labelled as retweets.

Table 3 shows details of data cleaning.

\footnotetext{
${ }^{3} 59 \%$ of the statuses collected were tagged as being in English.
} 
Table 3. Details of Data Cleaning

\begin{tabular}{lrrrrrr}
\hline $\begin{array}{l}\text { Keyword/ } \\
\text { Project }\end{array}$ & $\begin{array}{l}\text { Number } \\
\text { sampled }\end{array}$ & Irrelevant & $\begin{array}{c}\text { Not } \\
\text { English }\end{array}$ & Robots & Duplicates & $\begin{array}{c}\text { Number } \\
\text { after } \\
\text { cleaning }\end{array}$ \\
\hline Joomla & 1,000 & 1 & 1 & 323 & 27 & 650 \\
TYPO3 & 1,000 & 0 & 3 & 45 & 1 & 951 \\
SilverStripe & 1,000 & 0 & 3 & 121 & 23 & 854 \\
Drupal & 1,000 & 1 & 4 & 147 & 9 & 839 \\
Xoops & 676 & 9 & 39 & 164 & 55 & 417 \\
Plone & 1,000 & 20 & 7 & 196 & 5 & 772 \\
RoR & 1,000 & 218 & 7 & 78 & 24 & 673 \\
PHP & 1,000 & 6 & 8 & 297 & 20 & 674 \\
Apache & 1,000 & 565 & 5 & 148 & 6 & 277 \\
Mozilla & 1,000 & 236 & 34 & 5 & 0 & 725 \\
OpenOffice & 1,000 & 0 & 18 & 31 & 49 & 902 \\
libreoffice & 1,000 & 0 & 33 & 266 & 18 & 696 \\
\hline Sum & 11,676 & 1,056 & 162 & 1,821 & 237 & 8,430 \\
\hline
\end{tabular}

\subsection{Data Coding}

Ehrlich and Shami [6], building on work by Java et al. [7] and by Zhao and Rosson [8], proposed that microblog posts can be sorted into six categories. This study employed Ehrlich and Shami's scheme, which is introduced below along with examples from our data. User names have been changed.

1. Status (giving details of the poster's current activities):

"Doing some module troubleshooting on the @Xoops_forums \#XOOPS \#imAwesome :P"

2. Provide information (sharing information/URLs, reporting news)

"Get an overview and demonstration of \#Acquia Search: http://t.co/ 9I352KgY \#Drupal"

3. Directed posts (addressed to one or more other users):

"@userA Ah, well on TYPO3 Sonar you can't - that's decided by the profile. If you want to change it you'd need your own Sonar install imo."

4. Retweets:

"RT @userB: PC World calls out \#Plone as one of 10 award-winng apps to try: http://t.co/WOK9mU4Y"

5. Ask question:

"CodeKit users: can I selectively add files from a project? I really don't need it to monitor my entire SilverStripe install, just templates." 
6. Directed with question.

“@userC im just getting into Web Development, but i dont if it would be more begginer firiendly to learn PHP or RoR, help!?"

It was not always easy to decide which category a post should fall into; making a distinction between status and provide information proved particularly difficult, e.g.

"In thinking caps on Mozilla writeable society session. A few mins, great minds and awesome ideas. See 'em here: http://t.co/dHk1zVSk \#pdf12"

In such cases, we put the Tweet into the current status category, which we broadened to include notices and reports of events happening on the day of posting e.g.

"Great RoR Meetup tonight at @manilla office with @userD and lots of swearing. Good times."

The coding was carried out by the author alone; clearly it would have been better to have three or more coders in order to reduce error and bias. Table 4 shows the numbers of statuses in each category, and Figure 1 displays the same data as proportions.

We also broke down the large "provide information" category into domainspecific subcategories. Table 5 and Figure 2 show the numbers and proportions of subcategories respectively.

\section{Results and Analysis}

\subsection{Status Updates versus Information Provision}

Do OSS-related statuses follow general Twitter usage in concentrating on the user's current activities, or do they tend to provide more general information?

Motivation In their study of general Twitter users [9], Naaman et al. used cluster analysis to suggest that $80 \%$ of the users sampled were "Meformers", who mostly tweeted about what they were doing, and only $20 \%$ were "Informers" forwarding information. In contrast, Ehrlich and Shami [6] found that about $10 \%$ of tweets sent by their regular users in IBM were about current status, compared with just under $30 \%$ that were providing information. We can therefore hypothesize that, given a more professional context, OSS-related tweets will have a higher proportion of information provision than "Me now" content. However, this is not to deny the potential value of "Me now" posts as a lubricant maintaining easy communication between participants in OSS projects.

Results Percentages of current status updates were between $0.9 \%$ and $8.5 \%$ (see Table 4 and Figure 1), while those providing information accounted for between $36 \%$ and $73 \%$ of each sample. 
Table 4. Numbers of Statuses in Each Category

\begin{tabular}{lrrrrrr|r}
\hline $\begin{array}{l}\text { Keyword } \\
\text { project }\end{array}$ & $\begin{array}{c}\text { Ask } \\
\text { question }\end{array}$ & Directed & $\begin{array}{c}\text { Directed } \\
\text { with } \\
\text { question }\end{array}$ & $\begin{array}{c}\text { Provide } \\
\text { info }\end{array}$ & Retweet & $\begin{array}{c}\text { Current } \\
\text { status }\end{array}$ & Sum \\
\hline Joomla & 3 & 28 & 7 & 455 & 146 & 11 & 650 \\
TYPO3 & 57 & 75 & 23 & 321 & 419 & 56 & 951 \\
SilverStripe & 9 & 52 & 9 & 381 & 371 & 32 & 854 \\
Drupal & 12 & 71 & 12 & 435 & 272 & 37 & 839 \\
Xoops & 2 & 5 & 2 & 240 & 163 & 5 & 417 \\
Plone & 11 & 42 & 8 & 277 & 400 & 34 & 772 \\
RoR & 13 & 92 & 13 & 417 & 109 & 29 & 673 \\
PHP & 6 & 28 & 3 & 455 & 176 & 6 & 674 \\
Apache & 8 & 12 & 4 & 151 & 93 & 9 & 277 \\
Mozilla & 3 & 30 & 5 & 439 & 224 & 24 & 725 \\
OpenOffice & 27 & 132 & 21 & 363 & 285 & 74 & 902 \\
libreoffice & 34 & 119 & 27 & 259 & 198 & 59 & 696 \\
\hline Sum & 185 & 686 & 134 & 4,193 & 2,856 & 376 & 8,430 \\
\hline
\end{tabular}

Table 5. Subcategories of "Provide Information" Statuses

\begin{tabular}{lrrrrrrrr|r}
\hline $\begin{array}{l}\text { Keyword/ } \\
\text { project }\end{array}$ & Book & $\begin{array}{c}\text { Code } \\
\text { release }\end{array}$ & Event & $\begin{array}{c}\text { Documen- } \\
\text { tation }\end{array}$ & General & Jobs & $\begin{array}{c}\text { News- } \\
\text { letter }\end{array}$ & Security & Sum \\
\hline Joomla & 8 & 42 & 9 & 10 & 208 & 172 & 1 & 5 & 455 \\
TYPO3 & 3 & 40 & 61 & 10 & 182 & 10 & 13 & 2 & 321 \\
SilverStripe & 3 & 86 & 39 & 18 & 175 & 18 & 0 & 42 & 381 \\
Drupal & 14 & 34 & 56 & 52 & 160 & 117 & 0 & 2 & 435 \\
Xoops & 1 & 24 & 0 & 101 & 85 & 15 & 4 & 10 & 240 \\
Plone & 18 & 37 & 78 & 14 & 106 & 9 & 0 & 15 & 277 \\
RoR & 0 & 3 & 9 & 10 & 67 & 328 & 0 & 0 & 417 \\
PHP & 11 & 10 & 3 & 21 & 87 & 312 & 0 & 11 & 455 \\
Apache & 6 & 22 & 5 & 22 & 72 & 12 & 0 & 12 & 151 \\
Mozilla & 0 & 28 & 9 & 6 & 384 & 0 & 0 & 12 & 439 \\
OpenOffice & 8 & 36 & 4 & 8 & 288 & 4 & 0 & 15 & 363 \\
libreoffice & 0 & 32 & 5 & 24 & 183 & 2 & 0 & 13 & 259 \\
\hline Sum & 72 & 394 & 278 & 296 & 1,997 & 999 & 18 & 139 & 4,193 \\
\hline
\end{tabular}

The two office applications both show a higher proportion of current status posts than the other projects. Reading the statuses did indeed suggest a lot of general users posting tweets along the lines of

"man im tryna type this paper but for whatever reason my OpenOffice is gone!"

This supports the hypothesis that statuses written by professionals related to their work tend to be more information providing than "Me now"-ish.

A number of factors help to explain the higher proportions of information providing Tweets in our samples than those found by Ehrlich and Shami. First, 


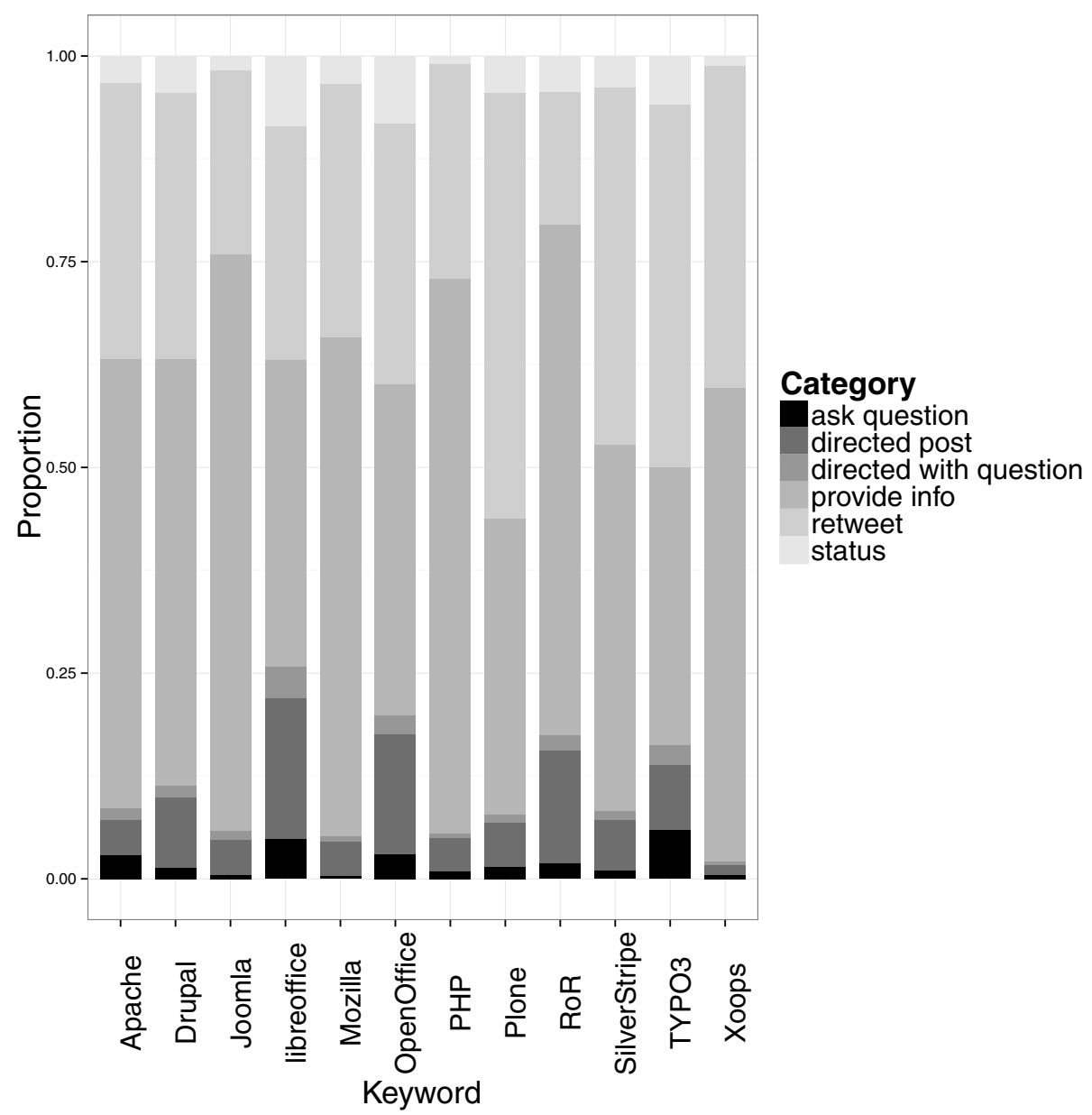

Fig. 1. Categories of Twitter Status

despite excluding many automatically generated job-related posts at the data cleaning stage, 1035 job-related posts remain in the "provide information" category; it is unlikely that Ehrlich and Shami's subjects would be sending many such posts. Second, the "provide information" category includes advertisements for online resources such as articles, books or software, which are also less likely to be sent by Ehrlich and Shami's subjects. Third, Naaman et al. found that tweets sent from mobile terminals are more likely than those sent from computers to be "Me now" rather than "provide information", and we can expect that most of the users and developers of the projects studied will be working on computers. Fourth, Naaman et al. also found that women rather than men are more likely to post "Me now" statuses, and given the high proportion of male participation in most open source software projects this may be having some effect. 


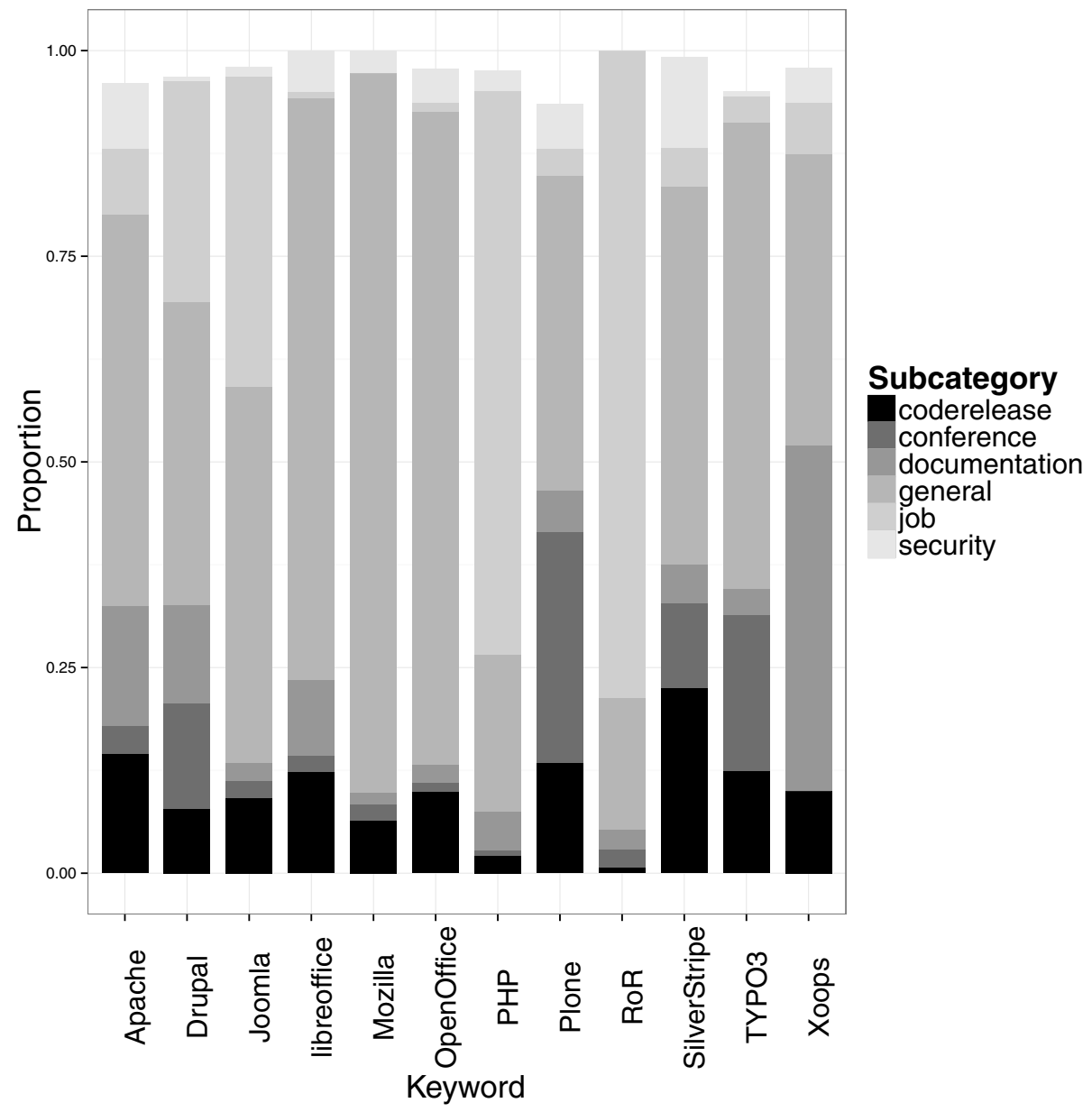

Fig. 2. Main subcategories of "provide information" statuses

\subsection{Event-Driven Traffic}

How much activity is prompted by events, both off- and online?

Motivation. Research on microblogging has shown that much activity occurs around events, particularly but not confined to offline events such as conferences [10, 11]. Vega et al. 12] found that conference participants tweeted more than usual in the week of the event. Conferences, and smaller events such as sprints and meetups, are the site of intense discussions between participants on all aspects of their project, so we can expect greater Twitter activity to exchange information with other participants and also to communicate what is happening.

Results. Table 5 and Figure 2 show the numbers and proportions of event-related tweets for the 12 project keywords. 
Proportions of event-related tweets are low for all keywords except TYPO3 and Plone. In the case of TYPO3, there was something of a spike in activity around the conference held in Stuttgart in early October 2012, while for Plone there was a more pronounced concentration of tweets around the conference held in Arnhem in the same month. While it is not easy to distinguish current status and provide information categories concerning events, for neither project did tweets sharing current status e.g.

"home and awake after a cool (exhausting) \#T3CON12DE - now let's rock \#TYPO3, \#NEOS and everything like we rocked this conference!"

predominate, suggesting that communication is more focused on providing information about the conference to participants and non-participants e.g.

"Thanks for attending our talk on \#TYPO3 and \#TYPO3Neos at \#drupalhagen. Our slides are available here https://t.co/j6RJolxx."

It is interesting to note that for both TYPO3 and Plone, when we exclude jobrelated statuses, we also find that a higher proportion of statuses for these two projects mention "community" than is the case with other projects 4 Only 4 out of the 35 and 7 out of the 56 statuses for Plone and TYPO3 respectively that mentioned community were event-related, e.g.

"The \#Plone community is just fucking crazy (in a good way). Prost!!!! \#beersprint"

Therefore this does not seem to be merely a case of people tweeting about community when surrounded by their fellow developers and users. Further investigation is required to clarify whether this high proportion of event-related statuses and mentions of community is a coincidence, and if not then what is the relationship between the two. While it goes without saying that talking about community does not create one, the spontaneous nature of Twitter makes it a promising medium to explore how open source users and developers think and feel about their projects.

\subsection{Rival Products}

To what extent do statuses mention non-code related factors such as rival products?

Motivation. In their study of GNOME mailing lists, Shibab et al. [13] found that external factors, and particularly the emergence of rival products, played a significant role in shaping discussions among developers. They used these external developments to explain a decline in the market share of the Evolution

${ }^{4}$ We choose to ignore the large number of mentions of "community" for PHP because 24 of the 27 mentions are due to retweeting of a status praising one company's community engagement. The lack of comments on the retweets suggested an advertising campaign. 
Table 6. Frequently occuring words in non-job related statuses

\begin{tabular}{|c|c|c|c|}
\hline $\begin{array}{l}\text { Project/ } \\
\text { keyword }\end{array}$ & $\begin{array}{c}\text { number of } \\
\text { statuses }\end{array}$ & word & $\begin{array}{l}\text { number of } \\
\text { occurrences }\end{array}$ \\
\hline \multirow[t]{2}{*}{ Joomla } & 561 & wordpress & 106 \\
\hline & & drupal & 31 \\
\hline TYPO3 & 979 & (none) & \\
\hline Silverstripe & 921 & wordpress & 38 \\
\hline \multirow{2}{*}{ Drupal } & 765 & wordpress & 75 \\
\hline & & joomla & 44 \\
\hline Xoops & 525 & (none) & \\
\hline Plone & 950 & wordpress & 36 \\
\hline RoR & 368 & php & 23 \\
\hline \multirow[t]{5}{*}{$\overline{\mathrm{PHP}}$} & 374 & apache & 110 \\
\hline & & mysql & 83 \\
\hline & & ruby & 30 \\
\hline & & rails & 30 \\
\hline & & magento & 23 \\
\hline \multirow[t]{2}{*}{ Apache } & 406 & mysql & 137 \\
\hline & & openoffice & 30 \\
\hline \multirow[t]{5}{*}{ Mozilla } & 729 & google & 75 \\
\hline & & chrome & 45 \\
\hline & & windows & 41 \\
\hline & & ipad & 32 \\
\hline & & microsoft & 28 \\
\hline \multirow[t]{4}{*}{ OpenOffice } & $\overline{923}$ & libreoffice & 94 \\
\hline & & excel & 93 \\
\hline & & word & 68 \\
\hline & & microsoft & 42 \\
\hline \multirow[t]{2}{*}{ libreoffice } & 946 & openoffice & 68 \\
\hline & & office & 68 \\
\hline
\end{tabular}

mail client as rival products emerged. We can expect that a similar analysis of Twitter activity will show which products are perceived as rivals by those within and without open source projects. These findings, particularly if they could be tracked over years rather than months, would help to explain design decisions and shifts in market share.

Results. We excluded job-related statuses, then counted the occurrences of words in the text of statuses for each keyword/project. Table 6 shows a selection of the technology-related words appearing in the top 50 most commonly used words for each project, along with the number of statuses analyzed. Note that the number of occurrences can be greater than the number of statuses because project names are often used more than once in a single tweet. Some of the words are components or closely related to the projects and some are rival products.

Of the CMSes, statuses regarding all except TYPO3 and Xoops mention rival products, predominantly Wordpress. It would be worthwhile to follow this up with a longitudinal study to establish whether Wordpress is gaining in its 
position as the chief rival to most open source CMSes. Tweets about the office products, as expected, mention each other and Microsoft Office. It is also interesting to see that statuses on $\mathrm{PhP}$ and Ruby on Rails both make significant mention of each other.

\section{Threats to Validity and Topics for Further Research}

One limitation of this study is that it compares proportions of different kinds of Twitter use across projects/keywords based on samples of similar size, while the absolute numbers of statuses for the different keywords differ greatly. Therefore, while we can conclude that e.g. a higher proportion of Silverstripe-related tweets than Apache-related tweets are concerned with events, that does not mean that Apache users and developers do not make equally active use of Twitter with regard to events, while also using Twitter more actively to e.g. provide links to documentation.

It would be desirable to increase the sample size for each project in order to increase the reliability of the data. It would also be better if the sample came from a full twelve-month period because many projects have large conferences once a year, and the current seven-month period risks excluding some events.

Not all statuses containing those keywords during the period were collected, for two reasons. First, the Twitter Search API does not guarantee to return all statuses for a given period. Second, it was not possible to ensure that the data collection script ran uninterrupted for the entire period. When such interruptions occurred, and the volume of posts was high, due to the limitation placed on the number of results returned from Twitters Search API (1500 in this case), it was not possible to collect all the statuses posted since the previous collection. These gaps might introduce distortions into the findings, for example if they coincide with a major conference or code release related to a particular project, thus missing a spike in microblogging activity. However, the long period of data collection can be expected to reduce the impact and perhaps to even out such distortions. The incompleteness of the data would also be a problem if the study were aiming to do a network analysis of Twitter-based communication in OSS projects, as there would be many missing directed messages and replies. However, as our purpose here is merely to analyze the content of individual statuses, this is not an issue for the current research 5

In retrospect, it would have been desirable to include Wordpress, one of the most popular open source CMSes. Unfortunately Twitter's search API does not allow statuses more than a few days old to be collected 6

The study could also be improved by obtaining information about numbers of followers and giving greater weight to posts that are more read.

\footnotetext{
${ }^{5}$ In addition, gathering only statuses that contain particular keywords would not capture all the directed Tweets sent between any given set of users.

${ }^{6}$ Some commercial services offer to retrieve historical Tweets.
} 


\section{References}

1. Boehringer, M., Richter, A.: Adopting enterprise 2.0: A case study on microblogging. Mensch \& Computer 2009: Grenzenlos frei (2009)

2. Reinhardt, W.: Communication is the key-support durable knowledge sharing in software engineering by microblogging. In: Proc. of the SENSE Workshop, Software Engineering within Social Software Environments (2009)

3. Riemer, K., Richter, A., Bohringer, M.: Enterprise microblogging. Business \& Information Systems Engineering 2(6), 391-394 (2010)

4. Guenther, O., Krasnova, H., Riehle, D., Schoendienst, V.: Modeling microblogging adoption in the enterprise. In: Americas Conference on Information Systems (AMCIS) 2009 Proceedings, vol. 544 (2009)

5. Riemer, K., Richter, A.: Tweet inside: Microblogging in a corporate context. In: Proceedings of the 23rd Bled eConference, pp. 1-17 (2010)

6. Ehrlich, K., Shami, N.: Microblogging inside and outside the workplace. In: ICWSM 2010 (2010)

7. Java, A., Song, X., Finin, T., Tseng, B.: Why we twitter: understanding microblogging usage and communities. In: Proceedings of the 9th WebKDD and 1st SNAKDD 2007 Workshop on Web Mining and Social Network Analysis, pp. 56-65 (2007)

8. Zhao, D., Rosson, M.B.: How and why people twitter: the role that micro-blogging plays in informal communication at work. In: Proceedings of the ACM 2009 International Conference on Supporting Group Work, pp. 243-252 (2009)

9. Naaman, M., Boase, J., Lai, C.: Is it really about me?: message content in social awareness streams. In: Proceedings of the 2010 ACM Conference on Computer Supported Cooperative Work, pp. 189-192 (2010)

10. Vega, E.: Communities of Tweeple: How Communities Engage with Microblogging When Co-located. PhD thesis, Virginia Polytechnic Institute and State University (2011)

11. Ebner, M., Mühlburger, H., Schaffert, S., Schiefner, M., Reinhardt, W., Wheeler, S.: Getting granular on twitter: Tweets from a conference and their limited usefulness for non-participants. In: Reynolds, N., Turcsányi-Szabó, M. (eds.) KCKS 2010. IFIP AICT, vol. 324, pp. 102-113. Springer, Heidelberg (2010)

12. Vega, E., Parthasarathy, R., Torres, J.: Where are my tweeps?: Twitter usage at conferences. Paper, Personal Information Management class, Virginia Polytechnic Institute and State University (June 1) (2010), http://www.socialcouch.com/demos/final_paper_twitter.pdf

13. Shibab, E., Bettenburg, N., Adams, B., Hassan, A.E.: On the central role of mailing lists in open source projects: An exploratory study. In: Proceedings of the 3rd International Workshop on Knowledge Collaboration in Software Development, KCSD, Kyoto, Japan (November 2009) 